\title{
Estrabismo paralítico: revisão de 24 anos da Santa Casa de São Paulo
}

\author{
Paralytic strabismus: review of 24 years at "Santa Casa de São Paulo"
}

\author{
Gustavo Bueno de Camargo ${ }^{1}$ \\ Wilson Takashi Hida ${ }^{2}$ \\ Mauro Goldchmit ${ }^{3}$ \\ Carlos Fumiaki Uesugui ${ }^{4}$ \\ Carlos Ramos de Souza-Dias ${ }^{5}$
}

\begin{tabular}{|l|}
\hline RESUMO \\
\hline Objetivo: Avaliar a incidência, etiologia e evolução dos estrabismos \\
paralíticos ou paréticos. Métodos: Foram selecionados retrospectiva- \\
mente 519 prontuários de pacientes com paresia ou paralisia isolada dos \\
músculos inervados pelos III, IV ou VI nervos cranianos, a partir de \\
11.000 prontuários da Seção de Motilidade Extrínseca Ocular do Depar- \\
tamento de Oftalmologia da Santa Casa de São Paulo de janeiro de 1980 \\
a outubro de 2004. Foram estudados: o nervo craniano acometido, o olho \\
acometido, o sexo, os fatores etiológicos e a evolução desses pacientes. \\
Resultados: Dos 519 pacientes, 17,1\% foram casos congênitos e 82,9\% \\
foram adquiridos. O nervo craniano mais afetado foi o VI (49,7\%). Os \\
pacientes do sexo masculino foram mais acometidos, com 58,1\% dos \\
casos. A etiologia traumática foi a mais freqüente nos casos de paresia \\
ou paralisia de III (43,0\%), IV (52,4\%) e VI (48,8\%) nervos cranianos. Os \\
pacientes evoluíram mais freqüentemente para cirurgia nos três grupos: \\
III nervo (42,9\%), IV nervo (73,2\%)e VI nervo (43,2\%). Conclusão: O VI \\
nervo craniano foi o mais freqüentemente acometido e o fator etiológico \\
mais importante foi o traumatismo, dados esses que coincidem com os \\
encontrados na literatura.
\end{tabular}

Descritores: Estrabismo/epidemiologia; Estrabismo/etiologia; Nervos cranianos/fisiopatologia

\section{INTRODUÇÃO}

Os estrabismos paralíticos e paréticos são quadros caracterizados pela fraqueza de um ou mais músculos oculomotores inervados pelo(s) nervo(s) craniano(s) afetado(s), que resulta em um desvio incomitante que pode necessitar de tratamento clínico, cirúrgico ou apresentar regressão espontânea.

Muitos estudos foram realizados com a finalidade de analisar as paralisias ou paresias dos III, IV e VI nervos cranianos e alguns se tornaram clássicos $^{(1-3)}$. Na literatura, o nervo craniano mais acometido é o VI, como observado diversos estudos, com frequiência de $40,9 \%$ a $55 \%{ }^{(1-4)}$.

Com relação ao sexo dos pacientes com estrabismo parético ou paralítico, em outro estudo encontrou $54,3 \%$ do sexo masculino e $45,7 \%$ do feminino ${ }^{(5)}$.

Alguns estudos encontraram $45,1 \%$ de casos com o olho direito afetado, $46,7 \%$ com o olho esquerdo e $8,2 \%$ com comprometimento bilateral ${ }^{(5)}$.

Este estudo retrospectivo analisa a incidência, etiologia e evolução dos estrabismos paralíticos ou paréticos em pacientes atendidos na Seção de Motilidade Extrínseca Ocular do Departamento de Oftalmologia da Santa Casa de São Paulo. 
Em relação ao sexo dos pacientes, encontraram-se 58,1\% do masculino e $41,9 \%$ do feminino, o que coincide com o encontrado na literatura. Esse ligeiro predomínio do sexo masculino pode ser explicado pelo fato das causas traumáticas predominarem na amostra estudada.

Não houve discordância, quanto aos dados relativos ao olho afetado, entre nossa amostra e as da literatura, pois encontramos $49,5 \%$ de comprometimento do olho direito, $41,9 \%$ do olho esquerdo e $8,6 \%$ de comprometimento bilateral.

Em relação à paresia ou paralisia adquirida do III nervo craniano, $49 \%$ eram de etiologia traumática e $17,4 \%$ de causa indeterminada. Pesquisas realizadas ${ }^{(7)}$, apontaram $45,5 \%$ de casos congênitos. Já outros estudos ${ }^{(5)}$, encontraram maior frequência de casos indeterminados $(23,7 \%)$, seguidos de causa vascular $(19,8 \%)$. Outros estudos ${ }^{(8)}$, relataram freqüência de $40,5 \%$ de causa indeterminada e $29,2 \%$ de etiologia vascular. Essas diferenças podem ser explicadas pelo fato de ser muito grande o número de acidentes automobilísticos e agressões físicas no nosso país. A baixa ocorrência da etiologia vascular $(17,5 \%)$ é, provavelmente, devida à falta de diagnóstico ou de encaminhamento desses pacientes ao nosso Departamento.

Os casos de paresia ou paralisia adquirida do IV nervo craniano tiveram como causa mais freqüentes o traumatismo $(52,4 \%)$; os casos de causa indeterminada foram $30,5 \%$. Na literatura analisada encontraram ${ }^{(6)}$, entre os fatores etiológicos mais freqüentes, o traumatismo $(25,7 \%)$ e o vascular $(15,7 \%)$; os fatores indeterminados somaram $28,3 \%$. Outros estudos $^{(9)}$ relataram freqüência de $43,5 \%$ de casos congênitos, $38,8 \%$ de causa indeterminada e $7,6 \%$ de origem traumática. Outra pesquisa ${ }^{(10)}$, observou $39,5 \%$ de casos congênitos, $34,0 \%$ de causa traumática e $23,2 \%$ de origem indeterminada. Entre crianças, uma pesquisa ${ }^{(1)}$ constatou que $57,3 \%$ dos seus casos eram de causa indeterminada e $13,6 \%$ provenientes de traumatismo. O traumatismo adquire importância em nossa amostra, pelo mesmo motivo apresentado anteriormente.

Os pacientes portadores de paralisia do VI nervo craniano apresentaram fatores etiológicos com a seguinte frequência: traumatismo $48,8 \%$, indeterminados $23,1 \%$ e vascular $17,7 \%$, como as principais. Encontramos, na nossa amostra, 3 casos congênitos $(1,5 \%)$, o que é muito raro na literatura. Segundo a literatura analisada (12), "toda paralisia congênita de VI nervo craniano deve ser considerada Síndrome de Duane até prova em contrário". Entretanto, esses pacientes não apresentaram nenhuma característica clínica desta síndrome. Na amostra da revisão literária analisada ${ }^{(5)}$, a maioria dos casos tiveram causa indeterminada $(26,1 \%)$, seguidos das neoplasias $(21,3 \%)$ e dos traumatismos $(14,8 \%)$. Já outros trabalhos ${ }^{(8)}$, encontraram $41,2 \%$ de casos de origem indeterminada, $29,8 \%$ de causa vascular e 15,6\% traumáticos. Essas discrepâncias são provavelmente devidas às diferenças de cunho social e das dificuldades no processo de encaminhamento dos pacientes, assim como ocorreu nos casos das outras paralisias estudadas.

Neste estudo, observamos que 18,6\% dos casos adquiridos apresentaram regressão espontânea do quadro, 48,8\% foram submetidos a cirurgia e $18,6 \%$ dos casos ficaram sem seguimento. Na literatura ${ }^{(3,8)}$, observa-se que a regressão espontânea tem índice maior, pesquisas relataram uma freqüência de $48,3 \%$ e $66,6 \%$. Nesses estudos, os casos de etiologia vascular apresentaram o maior índice de regressão espontânea, grupo esse que apresentou baixa freqüência em nossa amostra. Além disso, o fato de termos perdido o seguimento de tantos pacientes, fato comum nos hospitais públicos, pode ter causado baixa da freqüência dessa regressão.

\section{ABSTRACT}

Purpose: To describe the incidence, etiologies and follow-up of patients with paralytic strabismus. Methods: Retrospective study of 519 strabismic patients with isolated III, IV or VI cranial nerve palsy of 11,000 charts of the Ocular Motility Section of the Department of Ophthalmology of "Faculdade de Ciências Médicas Santa Casa de São Paulo", Brazil, between January 1980 and October 2004. This study analyzed: the injured cranial nerve, affected eye, sex distribution, etiology and follow-up. Results: It was found that $17.1 \%$ of the cases were congenital and $82.9 \%$ acquired. The VI cranial nerve was the most frequently affected $(49.7 \%)$. The incidence was higher in males $(58.1 \%)$. Traumatism was the most common cause of III (43.0\%), IV (52.4\%) and VI (48.8\%) nerve palsy. Surgery was performed in the three groups: third nerve $(42.9 \%)$, fourth nerve $(73.2 \%)$ and sixth nerve $(43.2 \%)$. Conclusions: The sixth cranial nerve was the most frequently affected and the most common cause was traumatism, the same as observed in the literature.

Keywords: Strabismus/epidemiology; Strabismus/etiology; Cranial nerves/physiopathology

\section{REFERÊNCIAS}

1. Rucker CW. Paralysis of the third, fourth and sixth cranial nerves. Am J Ophthalmol. 1958;46(6):787-94.

2. Rucker CW. The causes of paralysis of the third, fourth and sixth cranial nerves. Am J Ophthalmol. 1966;61(5 Pt 2):1293-8.

3. Rush JA, Youge BR. Paralysis of cranial nerves III, IV, and VI. Cause and prognosis in 1,000 cases. Arch Ophthalmol. 1981;99(1):76-9.

4. Kodsi SR, Younge BR. Acquired oculomotor, trochlear, and abducent cranial nerve palsies in pediatric patients. Am J Ophthalmol. 1992;114(5):568-74.

5. Richard BW, Jones FR Jr, Younge BR. Causes and prognosis in 4,278 cases of paralysis of the oculomotor, trochlear, and abducens cranial nerves. Am J Ophthalmol. 1992;113(5):489-96.

6. Prieto-Diaz J, Souza-Dias C. In: Estrabismo $3^{a}$ ed. São Paulo: Roca; 1996; p. 377-442.

7. Avó DS, Gurgel DPA, Salomão SR. Estrabismo paralítico: Um estudo de 168 casos. Bol Bras Ortóp. 1986;12:28-34.

8. Abreu FJQ, Abreu Filho JMPQ, Abreu JMPQ. Paresias e paralisias dos nervos oculomotores. Estudo retrospectivo e prospectivo - período de 30 anos. Rev Bras Oftalmol. 1993;52(4):19-25.

9. Sydnor CF, Seaber JH, Buckley EG. Traumatic superior oblique palsies. Ophthalmology. 1982;89(2):134-8.

10. Von Noorden GK, Murray E, Wong SY. Superior oblique paralysis. A review of 270 cases. Arch Ophthalmol. 1986;104(12):1771-6.

11. Robb RM. Idiopathic superior oblique palsies in children. J Pedriatr Ophthalmol Strabismus. 1990;27(2):66-9.

12. Souza-Dias C. Congenital IV nerve palsy in Duane's syndrome until disproven. Binocular Vision Q. 1992;7(2):70-7. 